This is page no. 0 . 


\title{
Large Components of Bipartite Random Mappings
}

\author{
Jennie Hansen \\ Department of Actuarial Mathematics and Statistics, Heriot-Watt University, \\ Edinburgh EH14 4AS, UK
}

\section{Jerzy Jaworski}

Department of Discrete Mathematics, Adam Mickiewicz University, 60-769 Poznań, Poland

\section{Date: 27 March 2000}

\begin{abstract}
A bipartite random mapping $T_{K, L}$ of a finite set $V=V_{1} \cup V_{2},\left|V_{1}\right|=K$ and $\left|V_{2}\right|=L$, into itself assigns independently to each $i \in V_{1}$ its unique image $j \in V_{2}$ with probability $1 / L$ and to each $i \in V_{2}$ its unique image $j \in V_{1}$ with probability $1 / K$. We study the connected component structure of a random digraph $G\left(T_{K, L}\right)$, representing $T_{K, L}$, as $K \rightarrow \infty$ and $L \rightarrow \infty$. We show that, no matter how $K$ and $L$ tend to infinity relative to each other, the joint distribution of the normalized order statistics for the component sizes converges in distribution to the Poisson-Dirichlet distribution on the simplex $\nabla=\left\{\left\{x_{i}\right\}: \sum x_{i} \leq 1, x_{i} \geq x_{i+1} \geq 0\right.$ for every $\left.i \geq 1\right\}$.
\end{abstract}

\section{INTRODUCTION AND STATEMENT OF RESULTS}

A bipartite random mapping $T_{K, L}$ of a finite set $V=V_{1} \cup V_{2}, V_{1}=\{1,2, \ldots, K\}$ and $V_{2}=\{K+1, K+2, \ldots, K+L\}$, into itself assigns independently to each $i \in V_{1}$ its unique image $j \in V_{2}$ with probability $1 / L$ and to each $i \in V_{2}$ its unique image $j \in V_{1}$ with probability $1 / K$. The mapping $T_{K, L}$ can be represented by a random bipartite digraph $G\left(T_{K, L}\right)$ on a set of 'red' labelled vertices corresponding to the set $V_{1}$ and a set of 'blue' labelled vertices corresponding to the set $V_{2}$. So, for example, $G\left(T_{K, L}\right)$ has a directed edge from red vertex $i$ to blue vertex $j$ if and only if $T_{K, L}(i)=j$. Each connected component of $G\left(T_{K, L}\right)$ consists of a bipartite directed cycle with bipartite directed trees connected to the cycle. This model can be viewed as a 'two-dimensional' generalization of the uniform random mapping $T_{K}$ on a single set of vertices $V=\{1,2, \ldots, K\}$ where for each $i, j \in V$, $\operatorname{Pr}\left\{T_{K}(i)=j\right\}=1 / K$ and all assignments are independent. It is worth noting that 
although $T_{K, L}^{2}=T_{K, L} \circ T_{K, L}$ restricted to $V_{1}$ (say) is a random mapping from $V_{1}$ into $V_{1}$, such that for all $i, j \in V_{1}, \quad \operatorname{Pr}\left\{T_{K, L}^{2}(i)=j\right\}=1 / K, \quad T_{K, L}^{2} \neq T_{K}$ in distribution since, generally, the choices of the images are not independent, for example for all $i, j, v \in V_{1}$, $i \neq v$,

$$
\operatorname{Pr}\left\{T_{K, L}^{2}(i)=j \mid T_{K, L}^{2}(v)=j\right\}=\frac{1}{K}+\frac{1}{L}\left(1-\frac{1}{K}\right) .
$$

This dependence is strongest when $K$ and $L$ are of the same order.

Much is known (see for example the monograph by Kolchin [18]) about the component structure of the random digraph $G\left(T_{K}\right)$ which represents the uniform random mapping $T_{K}$. Aldous [1] has shown that the joint distribution of the normalized order statistics for the component sizes in $G\left(T_{K}\right)$ converges to the Poisson-Dirichlet(1/2) distribution. Also, if $Y_{k}$ denotes the number of components of size $k$ in $G\left(T_{K}\right)$ then the joint distribution of $\left(Y_{1}, Y_{2}, \ldots, Y_{b}\right)$ is close, in the sense of total variation, to the joint distribution of a sequence of independent Poisson random variables when $b=o(K / \log K)$ (see Arratia et.al. [3], [4]) and from this one obtains a functional central limit theorem for the component sizes (see also [10]). An analogous result for the order statistics of the cycle sizes in a random permutation was obtained by Vershik and Shmidt [22] and there is a Poisson approximation result and functional central limit theorem for the cycle sizes in a random permutation (see [2], [8]).

We are interested in determining to what extent such results hold for the component structure of $G\left(T_{K, L}\right)$ as $K, L \rightarrow \infty$. Of course, in the case of bipartite random mappings there are two parameters, so the limit laws obtained may depend on how fast $K$ and $L$ tend to infinity relative to each other. For example, if $L=K^{\alpha}$ with $\alpha>1$, then it is easy to verify by a first moment argument that, for example,

$$
\operatorname{Pr}\left\{G\left(T_{K, K^{\alpha}}\right) \text { has a component of size } 2\right\} \rightarrow 0 \text { as } K \rightarrow \infty,
$$

whereas the Poisson process approximation for uniform random mappings tells us that

$$
\operatorname{Pr}\left\{G\left(T_{K}\right) \text { has a component of size } 2\right\} \rightarrow 1-e^{-c} \quad \text { as } \quad K \rightarrow \infty
$$

where $c=\lim _{K \rightarrow \infty} E\left(Y_{2}\right)$. So there is no Poisson approximation result for the small component sizes of $G\left(T_{K, K^{\alpha}}\right)$ when $\alpha>1$. On the other hand, in this paper we show that the joint distribution of the normalized order statistics for the component sizes converges in distribution to the Poisson-Dirichlet(1/2) distribution no matter how $K$ and $L$ tend to infinity. Our result complements and extends earlier work by Jaworski [12],[13] on asymptotic limit laws for the total number of components in $G\left(T_{K, L}\right)$. To prove the Poisson-Dirichlet result, we first establish the limiting distribution for the size of the component containing a given vertex and this result may also be of independent interest.

In order to describe these results, we first give a convenient characterization of the Poisson-Dirichlet $(\theta)$ distribution which also yields a useful principle for establishing convergence in distribution to the Poisson-Dirichlet $(\theta)$ distribution on the simplex

$$
\nabla=\left\{\left\{x_{i}\right\}: \sum x_{i} \leq 1, x_{i} \geq x_{i+1} \geq 0 \quad \text { for every } \quad i \geq 1\right\} .
$$

Let $Z_{1}, Z_{2}, Z_{3}, \ldots$ be a sequence of i.i.d. random variables such that each $Z_{i}$ has a $\operatorname{Beta}(\theta)$ $(\theta>0)$ distribution with density $h(z)=\theta(1-z)^{\theta-1}$ on the unit interval $(0,1)$. Now define 
a transformation, $f$, of the sequence $\left(Z_{1}, Z_{2}, ..\right)$ such that $f\left(Z_{1}, Z_{2}, \ldots\right)=\left(W_{1}, W_{2}, W_{3}, ..\right)$ where $W_{1}=Z_{1}$ and $W_{n}=Z_{n}\left(1-Z_{1}\right)\left(1-Z_{2}\right) \cdots\left(1-Z_{n-1}\right)$ for $n>1$, and observe that $\left(W_{1}, W_{2}, \ldots\right) \in \tilde{\nabla}=\left\{\left\{x_{i}\right\}: x_{i} \geq 0, \sum x_{i} \leq 1\right\}$. Finally, define the map $g: \tilde{\nabla} \rightarrow \nabla$ such that $\left(g\left\{x_{i}\right\}\right)_{k}$ is the $k$ th largest term in the sequence $\left\{x_{i}\right\} \in \tilde{\nabla}$; then the random sequence $g \circ f\left(Z_{1}, Z_{2}, \ldots\right)=\left(Q_{1}, Q_{2}, Q_{3}, \ldots\right) \in \nabla$ has a Poisson-Dirichlet $(\theta)$ distribution. The following convergence principle is an important consequence of this characterization: suppose that $\left(Z_{1}(n), Z_{2}(n), \ldots\right)$ is a sequence of random variables such that the joint distribution of $\left(Z_{1}(n), Z_{2}(n), \ldots\right)$ converges to the joint distribution of the variables $\left(Z_{1}, Z_{2}, \ldots\right)$, then the joint distribution of the random sequence $g \circ f\left(Z_{1}(n), Z_{2}(n), \ldots\right)=\left(Q_{1}(n), Q_{2}(n), \ldots\right)$ converges to the Poisson-Dirichlet $(\theta)$ distribution. For further details see Hansen [11] and the references therein.

To see how the convergence principle can be applied to random bipartite mappings, we introduce some additional notation. Recall that $K$ denotes the number of 'red' vertices and $L$ denotes the number of 'blue' vertices, and $N=K+L$ is the total number of vertices in the random digraph $G\left(T_{K, L}\right)$. In addition, let $\mathcal{C}_{1}$ denote the component in $G\left(T_{K, L}\right)$ which contains the red vertex labelled 1. If $\mathcal{C}_{1} \neq G\left(T_{K, L}\right)$, then let $\mathcal{C}_{2}$ denote the component in $G\left(T_{K, L}\right) \backslash \mathcal{C}_{1}$ which contains the smallest red vertex; otherwise, set $\mathcal{C}_{2}=\emptyset$. For $t>2$ we define $\mathcal{C}_{t}$ iteratively: If $G\left(T_{K, L}\right) \backslash\left(\mathcal{C}_{1} \cup \ldots \cup \mathcal{C}_{t-1}\right) \neq \emptyset$, then let $\mathcal{C}_{t}$ denote the component in $G\left(T_{K, L}\right) \backslash\left(\mathcal{C}_{1} \cup \ldots \cup \mathcal{C}_{t-1}\right)$ which contains the smallest red vertex; otherwise, set $\mathcal{C}_{t}=\emptyset$. For $t \geq 1$, let $C_{t}=\left|\mathcal{C}_{t}\right|$ and define the sequence $\left(X_{1}(N), X_{2}(N), \ldots\right)=\left(X_{1}, X_{2}, \ldots\right)$ by

$$
X_{1}=\frac{C_{1}}{N}, X_{2}=\frac{C_{2}}{N-C_{1}}, \ldots, X_{t}=\frac{C_{t}}{N-C_{1}-C_{2}-\ldots-C_{t-1}}, \ldots
$$

where $X_{t}=0$ if $N-C_{1}-C_{2}-\ldots-C_{t-1}=0$. In Section 3 we show that for each $t \geq 1$ and $0<a_{i}<b_{i}<1, i=1,2, \ldots, t$

$$
\lim _{K \rightarrow \infty} \operatorname{Pr}\left\{a_{1}<X_{1} \leq b_{1}, a_{2}<X_{2} \leq b_{2}, \ldots, a_{t}<X_{t} \leq b_{t}\right\}=\prod_{i=1}^{t} \int_{a_{i}}^{b_{i}} \frac{d u}{2 \sqrt{1-u}}
$$

under the assumption that $L=L(K) \geq K$. We establish (1.1) by an inductive argument in Section 3. The first step in the induction is established in Section 2, where we prove

Theorem 1. Suppose that $L=L(K)$ and there is a positive constant $\eta$ such that $0<\eta K \leq L$ for all $K$, then for every $0<a<b<1$

$$
\operatorname{Pr}\left\{a N<C_{1} \leq b N\right\} \rightarrow \int_{a}^{b} \frac{d u}{2 \sqrt{1-u}} \quad \text { as } \quad K \rightarrow \infty
$$

To state our second result, let $D_{1}$ denote the size of the largest connected component in $G\left(T_{K, L}\right)$, let $D_{2}$ denote the size of the second largest component and so on. It is easy to check that

$$
g \circ f\left(X_{1}(N), X_{2}(N), \ldots\right)=\left(D_{1} / N, D_{2} / N, \ldots\right),
$$

so using the convergence principle for the Poisson-Dirichlet distribution, we obtain from $(1.1)$ 
Theorem 2. Let $N=K+L$ and $D_{1}, D_{2}, \ldots$ be as defined above, then

$$
\left(\frac{D_{1}}{N}, \frac{D_{2}}{N}, \ldots\right) \quad \stackrel{d}{\longrightarrow} \mathcal{P} \mathcal{D}(1 / 2) \quad \text { as } \quad L, K \rightarrow \infty
$$

where $\mathcal{P} \mathcal{D}(1 / 2)$ denotes the Poisson-Dirichlet(1/2) distribution on the simplex

$$
\nabla=\left\{\left\{x_{i}\right\}: \quad \sum x_{i} \leq 1, \quad x_{i} \geq x_{i+1} \geq 0 \quad \text { for every } i \geq 1\right\}
$$

\section{THE SIZE OF A CONNECTED COMPONENT}

We start with the exact joint probability distribution of the random variables $\left(R_{1}, B_{1}\right)$, where $R_{1}=R_{1}(i)$ is the number of red vertices (vertices from $V_{1}$ ) in the connected component $\mathcal{C}_{1}$ of $G\left(T_{K, L}\right)$ containing a given red vertex $i$ and $B_{1}=B_{1}(i)$ is the number of blue vertices (vertices from $V_{2}$ ) in this component. We will assume that $i=1$.

Lemma 1. For $k=0,1, \ldots, K-1$ and $l=1, \ldots, L$ we have

$$
\begin{gathered}
\operatorname{Pr}\left\{R_{1}=k+1, B_{1}=l\right\} \\
=\left(\begin{array}{c}
K-1 \\
k
\end{array}\right)\left(\begin{array}{c}
L \\
l
\end{array}\right)\left(\frac{k+1}{K}\right)^{l-1}\left(1-\frac{k+1}{K}\right)^{L-l}\left(\frac{l}{L}\right)^{k}\left(1-\frac{l}{L}\right)^{K-1-k} \\
\times \frac{1}{K L} \sum_{j=1}^{\min \{l, k+1\}} \frac{(l)_{j}}{l^{j}} \frac{(k+1)_{j}}{(k+1)^{j}}(k+l+1-j) .
\end{gathered}
$$

Proof. There are $\left(\begin{array}{c}K-1 \\ k\end{array}\right)$ ways to choose $k$ red vertices and $\left(\begin{array}{l}L \\ l\end{array}\right)$ ways to choose $l$ blue vertices which form the connected component containing the vertex "1". We have $(K-k-1)^{L-l}$ ways to map the remaining $L-l$ blue vertices into the remaining $K-k-1$ red vertices and $(L-l)^{K-k-1}$ ways to map $K-k-1$ red vertices into $L-l$ blue vertices. Finally, there are

$$
(k+1)^{l-1} l^{k} \sum_{j=1}^{\min \{l, k+1\}} \frac{(l)_{j}}{l^{j}} \frac{(k+1)_{j}}{(k+1)^{j}}(k+l+1-j)
$$

digraphs representing connected bipartite mappings on $k+1$ red and $l$ blue vertices [5], [12]. The result now follows immediately.

To prove Theorem 1 we need two more lemmas which are stated and proved below.

Lemma 2. Fix $\eta>0$, then for all $K, L>0$ such that $\eta K \leq L \leq K^{7 / 4}$ and for every $0<a<b<1$, there is a constant $C(a, b, \eta)$ which only depends on $a, b$, and $\eta$, such that

$$
\left|\operatorname{Pr}\left\{a N<C_{1} \leq b N\right\}-\int_{a}^{b} \frac{d x}{2 \sqrt{1-x}}\right| \leq \frac{C(a, b, \eta)}{K^{1 / 16}}
$$


where $N=K+L$.

Remark. Lemma 2 can be shown to hold more generally. In particular, it can be shown that if $\eta>0$ and $1 \leq \alpha<2$ are fixed, then for all $K>0$ and $L, \eta K \leq L \leq K^{\alpha}$,

$$
\left|\operatorname{Pr}\left\{a N<C_{1} \leq b N\right\}-\int_{a}^{b} \frac{d x}{2 \sqrt{1-x}}\right| \leq \frac{C(a, b, \eta, \alpha)}{K^{\zeta}}
$$

where $\zeta=\min \left\{\frac{1}{8}, \frac{1}{2}-\frac{\alpha}{4}\right\}$ and $C(a, b, \eta, \alpha)$ is a constant which only depends on $a, b, \eta$, and $\alpha$. However, for the proof of Theorem 1, it suffices to prove Lemma 2 in the case where $\alpha=7 / 4$. The restriction to this case also simplifies the proof of the lemma.

Proof. Throughout the proof $C(a, b, \eta)$ will denote any constant which may depend on $a, b$, and $\eta$ but which does not depend on $K$. Now fix $K>0$, fix $L$ such that $\eta K \leq L \leq K^{7 / 4}$, and suppose $m$ is such that $a N<m \leq b N$ where $N=K+L$. Let $x=m / N($ so $x \in(a, b])$ and let $[K x]$ and $[L x]$ be integers such that

$$
[K x]+[L x]=m \quad \text { and } \quad|[K x]-K x| \leq 1, \quad|[L x]-L x| \leq 1
$$

Then

$$
\operatorname{Pr}\left\{C_{1}=m\right\}=\sum_{-[L x]<j<[K x]} \operatorname{Pr}\left\{R_{1}=[K x]-j, B_{1}=[L x]+j\right\}
$$

where $R_{1}$ is the number of red vertices, and $B_{1}$ is the number of blue vertices in connected component $\mathcal{C}_{1}$. Now we split the above sum into two sums:

$$
\text { (i) } \sum_{|j| \leq \tau D(x)} \quad \text { (ii) } \sum_{|j|>\tau D(x)}
$$

where

$$
D(x)=\frac{K L}{K+L} \sqrt{\frac{x(1-x)}{K+L}} \leq C(a, b, \eta) \frac{K}{\sqrt{L}}
$$

and $\tau=K^{1 / 16}$.

In order to approximate the sums (2.3) we must investigate the joint distribution of $\left(R_{1}, B_{1}\right)$. Observe that the expression for $\operatorname{Pr}\left\{R_{1}=k+1, B_{1}=l\right\}$, given in Lemma 1 , can be split into three factors. The first factor

$$
\left(\begin{array}{c}
K-1 \\
k
\end{array}\right)\left(\frac{l}{L}\right)^{k}\left(1-\frac{l}{L}\right)^{K-1-k}
$$

represents the probability of the event of $k$ positive outcomes in the binomial distribution with parameters $K-1$ and $\frac{l}{L}$. Similarly, the second one

$$
\left(\begin{array}{l}
L \\
l
\end{array}\right)\left(\frac{k+1}{K}\right)^{l}\left(1-\frac{k+1}{K}\right)^{L-l}
$$


represents the probability of the event of $l$ positive outcomes in the binomial distribution with parameters $L$ and $\frac{k+1}{K}$. Approximations for both of these expressions with appropriate error bounds can be obtained from the proof of the de Moivre-Laplace Theorem (see Feller [9], p.182) in the case when $k+1=[K x]-j, l=[L x]+j$ and $|j| \leq \tau D(x)$. In particular,

$$
\left(\begin{array}{c}
L \\
l
\end{array}\right)\left(\frac{k+1}{K}\right)^{l}\left(1-\frac{k+1}{K}\right)^{L-l}=\frac{\exp \left(-\tilde{y}^{2} / 2\right)}{\sqrt{2 \pi L \frac{k+1}{K}\left(1-\frac{k+1}{K}\right)}} \cdot\left(1+\tilde{\rho}_{j}(x)\right)
$$

where $\tilde{y}$, the normalized deviation from the mean value, is given by

$$
\tilde{y}=\frac{[L x]+y D(x)-L([K x] / K-y D(x) / K)}{\sqrt{L([K x] / K-y D(x) / K)(1-[K x] / K+y D(x) / K)}}
$$

with $y=j / D(x)$. Now let

$$
\tilde{\Delta}_{j}(x)=[L x]+y D(x)-L([K x] / K-y D(x) / K),
$$

then

$$
\left|\tilde{\rho}_{j}(x)\right| \leq C(a, b, \eta) \cdot \max \left(\frac{\left|\tilde{\Delta}_{j}(x)\right|^{3}}{L^{2}}, \frac{\left|\tilde{\Delta}_{j}(x)\right|}{L}\right)
$$

and since

$$
\left|\tilde{\Delta}_{j}(x)\right| \leq C(a, b, \eta) L \tau D(x) / K \leq C(a, b, \eta) \tau \sqrt{L}
$$

we have

$$
\left|\tilde{\rho}_{j}(x)\right| \leq C(a, b, \eta) \cdot \frac{\tau^{3}}{\sqrt{L}} \leq C(a, b, \eta) K^{-5 / 16}
$$

We also have

$$
\tilde{y}=y \sqrt{\frac{L}{L+K}}+\tilde{\gamma}_{j}(x)
$$

where $\left|\tilde{\gamma}_{j}(x)\right| \leq C(a, b, \eta) K^{-1 / 8}$. Using the expression for $\tilde{y}$, we obtain

$$
\frac{\tilde{y}^{2}}{2}=\frac{y^{2} L}{2(L+K)}+y \sqrt{\frac{L}{L+K}} \cdot \tilde{\gamma}_{j}(x)+\frac{1}{2}\left(\tilde{\gamma}_{j}(x)\right)^{2}=\frac{y^{2} L}{2(L+K)}+\tilde{\varepsilon}_{j}(x),
$$

where

$$
\left|\tilde{\varepsilon}_{j}(x)\right| \leq C(a, b, \eta)\left|\tau \tilde{\gamma}_{j}(x)\right| \leq C(a, b, \eta) K^{-1 / 16} .
$$

So provided $a<x \leq b$ and $|j| \leq \tau D(x)$, we have

$$
\left(\begin{array}{l}
L \\
l
\end{array}\right)\left(\frac{k+1}{K}\right)^{l}\left(1-\frac{k+1}{K}\right)^{L-l}=\frac{\exp \left(\frac{-y^{2} L}{2(K+L)}+\tilde{\varepsilon}_{j}(x)\right)}{\sqrt{2 \pi L \frac{k+1}{K}\left(1-\frac{k+1}{K}\right)}} \cdot\left(1+\tilde{\rho}_{j}(x)\right)
$$

where

$$
\left|\tilde{\varepsilon}_{j}(x)\right| \leq C(a, b, \eta) K^{-1 / 16} \quad \text { and } \quad\left|\tilde{\rho}_{j}(x)\right| \leq C(a, b, \eta) K^{-5 / 16}
$$


Similarly one can show that

$$
\left(\begin{array}{c}
K-1 \\
k
\end{array}\right)\left(\frac{l}{L}\right)^{k}\left(1-\frac{l}{L}\right)^{K-1-k}=\frac{\exp \left(\frac{-y^{2} K}{2(K+L)}+\bar{\varepsilon}_{j}(x)\right)}{\sqrt{2 \pi(K-1) \frac{l}{L}\left(1-\frac{l}{L}\right)}} \cdot\left(1+\bar{\rho}_{j}(x)\right)
$$

where

$$
\left|\bar{\varepsilon}_{j}(x)\right| \leq C(a, b, \eta) \cdot K^{-1 / 8} \quad \text { and } \quad\left|\bar{\rho}_{j}(x)\right| \leq C(a, b, \eta) K^{-1 / 8}
$$

Finally, for $k+1=[K x]-j$ and $l=[L x]+j$ with $|j| \leq \tau D(x)$, one can show, as in the proof of Theorem 7 in [13], that the remaining factor in $\operatorname{Pr}\left\{R_{1}=k+1, B_{1}=l\right\}$ is given by

$$
\frac{1}{(k+1) L} \sum_{i=1}^{\min \{k+1, l\}} \frac{(l)_{i}(k+1)_{i}}{(l)^{i}(k+1)^{i}}(k+l+1-i)=\sqrt{\frac{\pi x(K+L)}{2 K L}} \cdot(1+\hat{\varepsilon}(x, j))
$$

where

$$
\hat{\varepsilon}(x, j) \leq \frac{C(a, b, \eta)}{K^{1 / 8}}
$$

Hence by (2.7)-(2.9) we obtain for $a<x=\frac{m}{N} \leq b$, and $|j| \leq \tau D(x)$

$$
\begin{aligned}
& \operatorname{Pr}\left\{R_{1}=[K x]-j, B_{1}=[L x]+j\right\}=\operatorname{Pr}\left\{R_{1}=[K x]-y D(x), B_{1}=[L x]+y D(x)\right\} \\
& =\frac{1}{K+L} \cdot \frac{1}{2 \sqrt{1-x}} \frac{(K+L) \sqrt{K+L}}{K L} \cdot \frac{1}{\sqrt{2 \pi x(1-x)}} \exp \left(\frac{-y^{2}}{2}\right) \cdot\left(1+\rho_{j}(x)\right)
\end{aligned}
$$

where $\left|\rho_{j}(x)\right| \leq C(a, b, \eta) \cdot K^{-1 / 16}$.

It follows that for $a<x=\frac{m}{N} \leq b$

$$
\begin{aligned}
& \sum_{|j| \leq \tau D(x)} \operatorname{Pr}\left\{R_{1}=[K x]-j, B_{1}=[L x]+j\right\} \\
= & \frac{1}{N} \cdot \frac{1}{2 \sqrt{1-x}} \sum_{|j| \leq \tau D(x)} \frac{1}{D(x)} \cdot \frac{\exp \left(-y^{2} / 2\right)}{\sqrt{2 \pi}} \cdot\left(1+\rho_{j}(x)\right) \\
= & \frac{1}{N} \cdot \frac{1}{2 \sqrt{1-x}} \cdot\left(1+\delta_{x}\right)
\end{aligned}
$$

where $\left|\delta_{x}\right| \leq C(a, b, \eta) \cdot K^{-1 / 16}$.

It remains to determine a bound for the second sum in (2.3). Since it is a 'two-sided' sum, we consider one side of the sum; the other case follows by similar calculations. The first step is to note that for all $k=0,1, \ldots, K-1$ and $l=1, \ldots, L$

$$
\operatorname{Pr}\left\{R_{1}=k+1, B_{1}=l\right\} \leq\left(\begin{array}{c}
L \\
l
\end{array}\right)\left(\frac{k+1}{K}\right)^{l}\left(1-\frac{k+1}{K}\right)^{L-l}
$$


since

$$
\left(\begin{array}{c}
K-1 \\
k
\end{array}\right)\left(\frac{l}{L}\right)^{k}\left(1-\frac{l}{L}\right)^{K-1-k} \leq 1
$$

and (see the end of the proof of Lemma 1)

$$
\frac{1}{(k+1) L} \sum_{j=1}^{\min \{l, k+1\}} \frac{(l)_{j}}{l^{j}} \frac{(k+1)_{j}}{(k+1)^{j}}(l+k+1-j)=\frac{l}{L} \operatorname{Pr}\left\{G\left(T_{k+1, l}\right) \text { is connected }\right\} \leq \frac{l}{L} .
$$

It follows that

$$
\begin{aligned}
& \sum_{j \geq \tau D(x)} \operatorname{Pr}\left\{R_{1}=[K x]-j, B_{1}=[L x]+j\right\} \\
\leq & \sum_{l \geq[L x]+\tau D(x)}\left(\begin{array}{l}
L \\
l
\end{array}\right)\left(\frac{m-l}{K}\right)^{l}\left(1-\frac{m-l}{K}\right)^{L-l}
\end{aligned}
$$

where $k+1=m-l$. Now the terms in the sum on the right hand side of (2.11) are binomial probabilities, but as we sum over the values of $l \geq[L x]+\tau D(x)$, the probability of 'success', $\frac{m-l}{K}$, changes as $l$ increases. Nevertheless, we claim that for all $l \geq[L x]+\tau D(x)$, we have

$$
\begin{aligned}
& \left(\begin{array}{c}
L \\
l+1
\end{array}\right)\left(\frac{m-l-1}{K}\right)^{l+1}\left(1-\frac{m-l-1}{K}\right)^{L-l-1} \\
\leq & \left(\begin{array}{c}
L \\
l
\end{array}\right)\left(\frac{m-l-1}{K}\right)^{l}\left(1-\frac{m-l-1}{K}\right)^{L-l} \\
\leq & \left(\begin{array}{c}
L \\
l
\end{array}\right)\left(\frac{m-l}{K}\right)^{l}\left(1-\frac{m-l}{K}\right)^{L-l} .
\end{aligned}
$$

The first inequality follows from the unimodality of the binomial distribution. To establish the second inequality, fix $l$ and define the function

$$
f_{l}(w)=\left(\tilde{x}-\frac{w}{K}\right)^{l}\left(1-\tilde{x}+\frac{w}{K}\right)^{L-l}
$$

where $\tilde{x}=\frac{[K x]}{K}$. It is not hard to show that $f_{l}^{\prime}(w)<0$ for $w \geq 0$, so if $l=[L x]+z$, where $z$ is some integer greater than $\tau D(x)$, then we have

$$
\left(\frac{m-l}{K}\right)^{l}\left(1-\frac{m-l}{K}\right)^{L-l}=f_{l}(z) \geq f_{l}(z+1)=\left(\frac{m-l-1}{K}\right)^{l}\left(1-\frac{m-l-1}{K}\right)^{L-l}
$$

since $m-l=[K x]-z$, and inequality (2.12) is established.

Now let $l_{0}=[L x]+\lfloor\tau D(x)\rfloor$ and $k_{0}+1=m-l_{0}=[K x]-\lfloor\tau D(x)\rfloor$ (where $\lfloor x\rfloor$ denotes the greatest integer less than $x$ ), then

$$
\begin{aligned}
& \sum_{l \geq[L x]+\tau D(x)}\left(\begin{array}{c}
L \\
l
\end{array}\right)\left(\frac{m-l}{K}\right)^{l}\left(1-\frac{m-l}{K}\right)^{L-l} \leq L \cdot\left(\begin{array}{c}
L \\
l_{0}
\end{array}\right)\left(\frac{m-l_{0}}{K}\right)^{l_{0}}\left(1-\frac{m-l_{0}}{K}\right)^{L-l_{0}} \\
& \quad \leq C(a, b, \eta) L \exp \left(\frac{-y^{2} L}{2(K+L)}\right) \leq C(a, b, \eta) K^{7 / 4} \exp \left(-K^{1 / 16}\right)
\end{aligned}
$$


where $y=\lfloor\tau D(x)\rfloor / D(x) \sim \tau=K^{1 / 16}$ and the second inequality above follows from (2.7). Therefore

$$
\sum_{j \geq \tau D(x)} \operatorname{Pr}\left\{R_{1}=[K x]-j, B_{1}=[L x]+j\right\} \leq C(a, b, \eta) K^{7 / 4} \exp \left(-K^{1 / 16}\right) .
$$

Similarly,

$$
\sum_{j \leq-\tau D(x)} \operatorname{Pr}\left\{R_{1}=[K x]-j, B_{1}=[L x]+j\right\} \leq C(a, b, \eta) K^{7 / 4} \exp \left(-K^{1 / 16}\right) .
$$

Combining the bounds (2.14)-(2.15) and the approximation (2.10), we obtain

$$
\operatorname{Pr}\left\{C_{1}=m\right\}=\frac{1}{N} \cdot \frac{1}{2 \sqrt{1-m / N}} \cdot\left(1+\delta_{x}\right)+\gamma_{m}
$$

where

$$
\left|\delta_{x}\right| \leq C(a, b, \eta) \cdot K^{-1 / 16}
$$

and

$$
\gamma_{m}=\sum_{|j| \geq \tau D(x)} \operatorname{Pr}\left\{R_{1}=[K x]-j, B_{1}=[L x]+j\right\} \leq C(a, b, \eta) K^{7 / 4} \exp \left(-K^{1 / 16}\right) .
$$

Hence

$$
\operatorname{Pr}\left\{a N<C_{1} \leq b N\right\}=\sum_{a N<m \leq b N} \frac{1}{N} \cdot \frac{1}{2 \sqrt{1-m / N}} \cdot\left(1+\delta_{x}\right)+\sum_{a N<m \leq b N} \gamma_{m}
$$

and it follows that

$$
\left|\operatorname{Pr}\left\{a N<C_{1} \leq b N\right\}-\int_{a}^{b} \frac{d x}{2 \sqrt{1-x}}\right| \leq \frac{C(a, b, \eta)}{K^{1 / 16}} .
$$

To approximate $\operatorname{Pr}\left\{a N<C_{1} \leq b N\right\}$ in the case where $L=L(K) \geq K^{7 / 4}$ we take an indirect approach. First, we recall (see [21]) that if $T_{K}$ is a uniform random mapping of $V_{1}$ into $V_{1}$, then for any $0<b<1$, we have

$$
\lim _{K \rightarrow \infty} \operatorname{Pr}\left\{\frac{C_{1}\left(T_{K}\right)}{K} \leq b\right\}=\int_{0}^{b} \frac{d x}{2 \sqrt{1-x}}
$$

where $C_{1}\left(T_{K}\right)$ denotes the size of the component which contains 1 in the directed graph $G\left(T_{K}\right)$ which represents $T_{K}$. Equation (2.16) also holds for the uniform model of random mappings without loops, $\hat{T}_{K}$, for which

$$
\operatorname{Pr}\left\{\hat{T}_{K}(i)=i\right\}=0 \quad \text { and } \operatorname{Pr}\left\{\hat{T}_{K}(i)=j\right\}=1 /(K-1) \quad \text { if } \quad i \neq j,
$$

for any $i, j \in V_{1}$, and all assignments are independent (see [15]). Now consider the random mapping $T^{2}=T_{K, L}^{2}=T_{K, L} \circ T_{K, L}$ restricted to the red vertices $V_{1}$. It is clear that $T^{2}$ is not 
a uniform random mapping on $V_{1}$, but if $L=L(K) \geq K^{7 / 4}$, then Lemma 3 below states that (2.16) holds with $C_{1}\left(T_{K}\right)$ (or $C_{1}\left(\hat{T}_{K}\right)$ ) replaced by $C_{1}\left(T^{2}\right)$, the size of the connected component containing 1 in $G\left(T^{2}\right)$, the digraph on the red vertices which represents $T^{2}$. In the proof of Theorem 1, we show that if $L=L(K) \geq K^{7 / 4}$, then the variables $\frac{C_{1}\left(T^{2}\right)}{K}$ and $\frac{C_{1}\left(T_{K, L}\right)}{N}$, where $N=K+L$, are 'close' with high probability and hence

$$
\operatorname{Pr}\left\{a N<C_{1} \leq b N\right\} \sim \int_{a}^{b} \frac{d x}{2 \sqrt{1-x}}
$$

Before proving Lemma 3, we define two random mapping models which are related to $\hat{T}_{K}$ and whose properties are exploited in the proof of the lemma. To construct the models (see also [14], [15] and [17]), we start with $G\left(\hat{T}_{K}\right)$, the random digraph which represents the uniform random mapping without loops $\hat{T}_{K}$ on $V_{1}$. To define the first model, fix $0 \leq M \leq K$, and let $G\left(\hat{T}_{K}, M\right)$ denote the random digraph obtained by deleting $K-M$ edges at random from $G\left(\hat{T}_{K}\right)$. To construct the second model, fix $0 \leq q \leq 1$ and let $Q \sim \operatorname{Bin}(K, q)$ be a binomial random variable which is independent of $\hat{T}_{K}$, and let $G\left(\hat{T}_{K} ; q\right)$ denote the random digraph obtained by choosing $Q$ vertices at random and deleting the edges in $G\left(\hat{T}_{K}\right)$ which are directed from the chosen vertices. Clearly, given $Q=K-M$, then $G\left(\hat{T}_{K} ; q\right)$ has the same distribution as $G\left(\hat{T}_{K}, M\right)$. Actually these two models of random mappings are related to each other in a very similar way as two classical models of random graphs $-G_{n, p}$ and $G_{n, M}$. One can easily adapt the methods which were used to study the relation between them (see [6] and [19]) to get the corresponding results about the equivalence of $G\left(\hat{T}_{K}, M\right)$ and $G\left(\hat{T}_{K} ; q\right)$. In particular, if $q \sim 1-M / K$ then many characteristics of the random digraphs $G\left(\hat{T}_{K}, M\right)$ and $G\left(\hat{T}_{K} ; q\right)$ have the same asymptotic distribution as $K \rightarrow \infty$.

To state the key property of both $G\left(\hat{T}_{K}, M\right)$ and $G\left(\hat{T}_{K} ; q\right)$, we need the following definition. A graph property $\mathcal{A}$ is decreasing (increasing) if given that a graph $G$ has $\mathcal{A}$, then every spanning subgraph (supergraph) of $G$ has this property also. We call such properties monotone. In a similar way one can define monotone properties of digraphs. Denote by $A(M)$ the number of digraphs (without loops) on $K$ vertices with $M$ vertices of out-degree 1 and with $K-M$ vertices of out-degree 0 , having a property $\mathcal{A}$. One can check that if $\mathcal{A}$ is a decreasing property, then

$$
(K-1)(K-M) A(M) \geq(M+1) A(M+1)
$$

(see [16] for a more general inequality). Notice that (2.17) implies that

$$
\begin{gathered}
\operatorname{Pr}\left\{G\left(\hat{T}_{K}, M\right) \text { has } \mathcal{A}\right\}=\frac{A(M)}{\left(\begin{array}{c}
K \\
M
\end{array}\right)(K-1)^{M}} \geq \frac{(M+1) A(M+1)}{(K-1)(K-M)\left(\begin{array}{l}
K \\
M
\end{array}\right)(K-1)^{M}} \\
=\frac{A(M+1)}{\left(\begin{array}{c}
K \\
M+1
\end{array}\right)(K-1)^{M+1}}=\operatorname{Pr}\left\{G\left(\hat{T}_{K}, M+1\right) \text { has } \mathcal{A}\right\}, \text { i.e., }
\end{gathered}
$$

The probability that the random digraph $G\left(\hat{T}_{K}, M\right)$ has a decreasing property $\mathcal{A}$ is a nonincreasing function of the parameter $M$. 
To prove the corresponding fact for $G\left(\hat{T}_{K} ; q\right)$ let us introduce the function

$$
f(z)=\operatorname{Pr}\left\{G\left(\hat{T}_{K} ; z\right) \text { has } A\right\}=\sum_{M=0}^{K} A(M) z^{K-M}\left(\frac{1-z}{K-1}\right)^{M}
$$

Then, since

$$
\begin{aligned}
& f^{\prime}(z)=\sum_{M=0}^{K-1} A(M)(K-M) z^{K-M-1}\left(\frac{1-z}{K-1}\right)^{M}-\sum_{M=1}^{K} A(M) \frac{M}{K-1} z^{K-M}\left(\frac{1-z}{K-1}\right)^{M-1} \\
& =\sum_{M=0}^{K-1} A(M)(K-M) z^{K-M-1}\left(\frac{1-z}{K-1}\right)^{M}-\sum_{M=0}^{K-1} A(M+1) \frac{M+1}{K-1} z^{K-M-1}\left(\frac{1-z}{K-1}\right)^{M},
\end{aligned}
$$

(2.17) implies that the first derivative of $f$ is non-negative for all $z, 0 \leq z \leq 1$, i.e.,

The probability that the random digraph $G\left(\hat{T}_{K} ; q\right)$ has a decreasing property $\mathcal{A}$ is a nondecreasing function of the parameter $q$.

Let $C_{1}(M)$ and $C_{1}(q)$ denote the size of the connected component which contains 1 in the random digraphs $G\left(\hat{T}_{K}, M\right)$ and $G\left(\hat{T}_{K} ; q\right)$, respectively. Notice, that ' $C_{1}(M) \leq x$ ' and ' $C_{1}(q) \leq x$ ' are decreasing properties. Therefore, for any $M_{1}, M_{2}$ and $x>0$, we have

$$
0 \leq M_{1} \leq M_{2} \leq K \quad \Rightarrow \quad \operatorname{Pr}\left\{C_{1}\left(M_{2}\right) \leq x\right\} \leq \operatorname{Pr}\left\{C_{1}\left(M_{1}\right) \leq x\right\}
$$

and for any $q_{1}, q_{2}$

$$
0 \leq q_{1} \leq q_{2} \leq 1 \Rightarrow \operatorname{Pr}\left\{C_{1}\left(q_{1}\right) \leq x\right\} \leq \operatorname{Pr}\left\{C_{1}\left(q_{2}\right) \leq x\right\}
$$

We also need the following result about the size $C_{1}(q)$ of the connected component of $G\left(\hat{T}_{K} ; q\right)$ containing a given vertex (see $\left.[15]\right)$ :

If $q \sqrt{K} \rightarrow 0$ as $K \rightarrow \infty$, then for every $0<b<1$,

$$
\lim _{K \rightarrow \infty} \operatorname{Pr}\left\{\frac{C_{1}(q)}{K} \leq b\right\}=\int_{0}^{b} \frac{d x}{2 \sqrt{1-x}} .
$$

We now proceed to prove

Lemma 3. Suppose that $L=L(K) \geq K^{7 / 4}$ for all $K>0$, then for any $0<b<1$,

$$
\lim _{K \rightarrow \infty} \operatorname{Pr}\left\{\frac{C_{1}\left(T^{2}\right)}{K} \leq b\right\}=\int_{0}^{b} \frac{d x}{2 \sqrt{1-x}} .
$$

Remark. Lemma 3 can also be shown to hold more generally. In particular, if $\alpha>3 / 2$ is fixed and $L=L(K) \geq K^{\alpha}$ for all $K>0$, then the conclusion of the lemma still holds. We prove the result for the case required in the proof of Theorem 1 . The proof of the lemma is also simplified by restricting to the case $\alpha=7 / 4$.

Proof. Throughout the proof $C$ will denote any constant which does not depend on $K$ (but which may depend on $b$ ). Now let $G\left(T^{2}\right)$ denote the random digraph on $V_{1}$ which represents the mapping $T^{2}=T_{K, L}^{2}$ and form the random digraph $G(\tilde{T})$ from $G\left(T^{2}\right)$ by 
deleting edges as follows. For each $v \in V_{1}$, delete the edge out of $v$ in $G\left(T^{2}\right)$ if $T^{2}(v)=v$ or if the vertex $T_{K, L}(v) \in V_{2}$ has in-degree greater than or equal to 2 in $G\left(T_{K, L}\right)$, the bipartite digraph which represents $T_{K, L}$, and let $G(\tilde{T})$ denote the resulting random digraph. Let $C_{1}(\tilde{T})$ denote the connected component in $G(\tilde{T})$ which contains vertex 1 . To prove the lemma, it suffices to show that if $L=L(K) \geq K^{7 / 4}$ for all $K>0$, then for every $0<b<1$

$$
\lim _{K \rightarrow \infty} \operatorname{Pr}\left\{\frac{C_{1}(\tilde{T})}{K} \leq b\right\}=\int_{0}^{b} \frac{d x}{2 \sqrt{1-x}}
$$

and

$$
\left|\frac{C_{1}(\tilde{T})}{K}-\frac{C_{1}\left(T^{2}\right)}{K}\right| \rightarrow 0 \quad \text { in probability as } K \rightarrow \infty .
$$

Let $Z$ denote the number of vertices in $G(\tilde{T})$ with out-degree 0 , then

$$
\begin{aligned}
\operatorname{Pr}\left\{\frac{C_{1}(\tilde{T})}{K} \leq b\right\} & =\sum_{j=0}^{K} \operatorname{Pr}\left\{\frac{C_{1}(\tilde{T})}{K} \leq b \mid Z=j\right\} \operatorname{Pr}\{Z=j\} \\
& =\sum_{j=0}^{K} \operatorname{Pr}\left\{\frac{C_{1}(K-j)}{K} \leq b\right\} \operatorname{Pr}\{Z=j\} .
\end{aligned}
$$

The second equality holds since given $Z=j$, the digraph $G(\tilde{T})$ has the same distribution as $G\left(\hat{T}_{K}, K-j\right)$. Now

$$
\begin{aligned}
E(Z) & =K \cdot \operatorname{Pr}\{\text { vertex } 1 \text { has out-degree } 0\}=K \cdot(1-\operatorname{Pr}\{\text { vertex } 1 \text { has out-degree } 1\}) \\
& =K \cdot\left(1-\operatorname{Pr}\left\{T^{2}(1) \neq 1 \text { and for every } v \in V_{1} \backslash\{1\}: T_{K, L}(v) \neq T_{K, L}(1)\right\}\right) \\
& =K\left(1-\left(1-\frac{1}{L}\right)^{K-1} \cdot \frac{K-1}{K}\right) \leq C K^{1 / 4}
\end{aligned}
$$

so $\operatorname{Pr}\left\{Z \geq K^{3 / 8}\right\} \leq C K^{-1 / 8}$. It follows from this bound and (2.18) that

$$
\begin{aligned}
\operatorname{Pr}\left\{\frac{C_{1}(\tilde{T})}{K} \leq b\right\} & \leq \sum_{j=0}^{\left\lfloor K^{3 / 8}\right\rfloor} \operatorname{Pr}\left\{\frac{C_{1}(K-j)}{K} \leq b \mid Z=j\right\} \operatorname{Pr}\{Z=j\}+C K^{-1 / 8} \\
& \leq \operatorname{Pr}\left\{\frac{C_{1}\left(K-\left\lfloor K^{3 / 8}\right\rfloor\right)}{K} \leq b\right\}+C K^{-1 / 8} .
\end{aligned}
$$

Next, set $q=K^{-9 / 16}$, and recall that $Q$, the number of edges deleted from $G\left(\hat{T}_{K}\right)$ to form $G\left(\hat{T}_{K} ; q\right)$, is a $\operatorname{Bin}(K, q)$ variable. So Chebyshev's inequality yields

$$
\operatorname{Pr}\left\{Q \leq K^{3 / 8}\right\} \leq C K^{-7 / 16} \text {. }
$$

This bound and (2.18) imply that

$$
\begin{aligned}
\operatorname{Pr}\left\{\frac{C_{1}(q)}{K} \leq b\right\} & \geq \sum_{j>K^{3 / 8}} \operatorname{Pr}\left\{\frac{C_{1}(q)}{K} \leq b \mid Q=j\right\} \operatorname{Pr}\{Q=j\} \\
& =\sum_{j>K^{3 / 8}} \operatorname{Pr}\left\{\frac{C_{1}(K-j)}{K} \leq b\right\} \operatorname{Pr}\{Q=j\} \\
& \geq \operatorname{Pr}\left\{\frac{C_{1}\left(K-\left\lfloor K^{3 / 8}\right\rfloor\right)}{K} \leq b\right\}\left(1-C K^{-7 / 16}\right) .
\end{aligned}
$$


Combining this inequality with (2.23) we obtain the upper bound

$$
\operatorname{Pr}\left\{\frac{C_{1}(\tilde{T})}{K} \leq b\right\} \leq \operatorname{Pr}\left\{\frac{C_{1}(q)}{K} \leq b\right\}+C K^{-1 / 8}
$$

and since $\lim _{K \rightarrow \infty} q \sqrt{K}=\lim _{K \rightarrow \infty} K^{-1 / 16}=0$, we have by $(2.20)$

$$
\limsup _{K \rightarrow \infty} \operatorname{Pr}\left\{\frac{C_{1}(\tilde{T})}{K} \leq b\right\} \leq \limsup _{K \rightarrow \infty} \operatorname{Pr}\left\{\frac{C_{1}(q)}{K} \leq b\right\}=\int_{0}^{b} \frac{d x}{2 \sqrt{1-x}} .
$$

To obtain a lower bound, we note that

$$
\operatorname{Pr}\left\{\frac{C_{1}(\tilde{T})}{K} \leq b\right\} \geq \operatorname{Pr}\left\{\frac{C_{1}(K)}{K} \leq b\right\}=\operatorname{Pr}\left\{\frac{C_{1}\left(\hat{T}_{K}\right)}{K} \leq b\right\} .
$$

It follows that

$$
\liminf _{K \rightarrow \infty} \operatorname{Pr}\left\{\frac{C_{1}(\tilde{T})}{K} \leq b\right\} \geq \lim _{K \rightarrow \infty} \operatorname{Pr}\left\{\frac{C_{1}\left(\hat{T}_{K}\right)}{K} \leq b\right\}=\int_{0}^{b} \frac{d x}{2 \sqrt{1-x}} .
$$

This inequality along with (2.24) establishes (2.21).

To establish (2.22), we first note that each connected component of the random digraph $G(\tilde{T})$ is either an oriented tree rooted at the vertex of out-degree 0 or it consists of exactly one oriented cycle together with oriented rooted trees. Denote by $F_{K}(\tilde{T})$ the set of vertices in all tree-components of $G(\tilde{T})$ and let $f_{K}(\tilde{T})=\left|F_{K}(\tilde{T})\right|$. Now suppose that

$$
\left\{v: v \in \mathcal{C}_{1}(\tilde{T})\right\} \cap F_{K}(\tilde{T})=\emptyset
$$

where $\mathcal{C}_{1}(\tilde{T})$ is the component in $G(\tilde{T})$ which contains 1 . In this case, $\mathcal{C}_{1}(\tilde{T})$ is not a tree-component and it follows that $\mathcal{C}_{1}(\tilde{T})$ was formed by cutting edges directed out of non-cyclical vertices in $\mathcal{C}_{1}\left(T^{2}\right)$, the component in $G\left(T^{2}\right)$ which contains 1 . Hence, in this case,

$$
C_{1}(\tilde{T}) \leq C_{1}\left(T^{2}\right) \leq C_{1}(\tilde{T})+f_{K}(\tilde{T}) .
$$

In particular, for any fixed $0<\rho<1,0<\beta<1, \varepsilon>0$, and all sufficiently large $K$, we have

$$
\left\{C_{1}(\tilde{T}) \geq \rho K, f_{K}(\tilde{T}) \leq K^{\beta}\right\} \subseteq\left\{\mathcal{C}_{1}(\tilde{T}) \cap F_{K}(\tilde{T})=\emptyset\right\} \subseteq\left\{\left|\frac{C_{1}(\tilde{T})}{K}-\frac{C_{1}\left(T^{2}\right)}{K}\right|<\varepsilon\right\}
$$

and hence

$$
\operatorname{Pr}\left\{C_{1}(\tilde{T}) \geq \rho K\right\}-\operatorname{Pr}\left\{f_{K}(\tilde{T})>K^{\beta}\right\} \leq \operatorname{Pr}\left\{\left|\frac{C_{1}(\tilde{T})}{K}-\frac{C_{1}\left(T^{2}\right)}{K}\right|<\varepsilon\right\} .
$$

To bound $\operatorname{Pr}\left\{f_{K}(\tilde{T}) \geq K^{\beta}\right\}$ we introduce some additional notation. If there exists an oriented path from $i$ to $j$ (including the null path from $i$ to $i$ ) in the digraph $G\left(\hat{T}_{K}\right)$, then $i$ is said to be a predecessor of $j$. For $A \subseteq V_{1}$, let $P_{\hat{T}_{K}}(A)$ denote the set of all predecessors in $G\left(\hat{T}_{K}\right)$ of elements in $A$, and in the special case where $A=\{1,2, \ldots, m\}$ for some $1 \leq m \leq K$, let $P_{\hat{T}_{K}}(m)=P_{\hat{T}_{K}}(\{1,2, \ldots, m\})$. Note that if $A_{1} \subset A_{2} \subset V_{1}$, then 
$P_{\hat{T}_{K}}\left(A_{1}\right) \subset P_{\hat{T}_{K}}\left(A_{2}\right)$. Moreover, the variables $\left|P_{\hat{T}_{K}}\left(A_{1}\right)\right|$ and $\left|P_{\hat{T}_{K}}\left(A_{2}\right)\right|$ have the same distribution whenever $\left|A_{1}\right|=\left|A_{2}\right|$. Now, given $A$ - the set of vertices $v \in V_{1}$ which have out-degree 0 in $G(\tilde{T}), f_{K}(\tilde{T})=\left|F_{K}(\tilde{T})\right|$ has the same distribution as $\left|P_{\hat{T}_{K}}(A)\right|$. So, in particular, $f_{K}(\tilde{T})$ can be studied as the total number of elements which are eventually infected in the inverse epidemic process $(I E P)$ on the digraph representing the uniform random mapping $\hat{T}_{K}$ (see [7] and [14]). It follows as in the derivation of (2.23) that

$$
\begin{aligned}
& \operatorname{Pr}\left\{f_{K}(\tilde{T}) \geq K^{\beta}\right\}=\sum_{j=0}^{K} \operatorname{Pr}\left\{f_{K}(\tilde{T}) \geq K^{\beta}|Z=| A \mid=j\right\} \operatorname{Pr}\{Z=j\} \\
& \leq \sum_{j=0}^{\left\lfloor K^{3 / 8}\right\rfloor} \operatorname{Pr}\left\{\left|P_{\hat{T}_{K}}(|A|)\right| \geq K^{\beta}|Z=| A \mid=j\right\} \operatorname{Pr}\{Z=j\}+C K^{-1 / 8} \\
& \leq \operatorname{Pr}\left\{\left|P_{\hat{T}_{K}}\left(\left\lfloor K^{3 / 8}\right\rfloor\right)\right| \geq K^{\beta}\right\}+C K^{-1 / 8}
\end{aligned}
$$

Now (see [14])

$$
E\left(\left|P_{\hat{T}_{K}}\left(\left\lfloor K^{3 / 8}\right\rfloor\right)\right|\right) \sim K^{3 / 8} \sqrt{K \pi / 2} \leq C \cdot K^{7 / 8},
$$

where $C$ is a constant which does not depend on $K$. Now let $\beta=15 / 16$, then by Markov's inequality we have

$$
\operatorname{Pr}\left\{\left|P_{\hat{T}_{K}}\left(\left\lfloor K^{3 / 8}\right\rfloor\right)\right| \geq K^{15 / 16}\right\} \leq C \cdot K^{-1 / 16}
$$

It follows from this bound and from inequality (2.26) that

$$
\begin{aligned}
\liminf _{K \rightarrow \infty} \operatorname{Pr}\left\{\left|\frac{C_{1}(\tilde{T})}{K}-\frac{C_{1}\left(T^{2}\right)}{K}\right| \leq \varepsilon\right\} & \geq \lim _{K \rightarrow \infty}\left(\operatorname{Pr}\left\{\frac{C_{1}(\tilde{T})}{K} \geq \rho\right\}-\operatorname{Pr}\left\{f_{K}(\tilde{T}) \geq K^{15 / 16}\right\}\right) \\
& =\int_{\rho}^{1} \frac{d x}{2 \sqrt{1-x}} .
\end{aligned}
$$

Letting $\rho \downarrow 0$, we obtain (2.22) and the lemma is proved.

We now proceed to prove Theorem 1.

Proof of Theorem 1. Fix $\varepsilon>0$, then we know from Lemma 2 that there exists $K_{\varepsilon}^{\prime}>0$ such that if $K \geq K_{\varepsilon}^{\prime}$ and $\eta K \leq L \leq K^{7 / 4}$, then

$$
\left|\operatorname{Pr}\left\{a<\frac{C_{1}}{N} \leq b\right\}-\int_{a}^{b} \frac{d x}{2 \sqrt{1-x}}\right|<\varepsilon .
$$

Now suppose that $L \geq K^{7 / 4}$ and choose $\delta>0$ such that

$$
\left|\int_{a-\delta}^{b+\delta} \frac{d x}{2 \sqrt{1-x}}-\int_{a}^{b} \frac{d x}{2 \sqrt{1-x}}\right| \leq \frac{\varepsilon}{4} \quad \text { and } \quad\left|\int_{a+\delta}^{b-\delta} \frac{d x}{2 \sqrt{1-x}}-\int_{a}^{b} \frac{d x}{2 \sqrt{1-x}}\right| \leq \frac{\varepsilon}{4} .
$$


Observe that

$$
\operatorname{Pr}\left\{a<\frac{C_{1}}{N} \leq b\right\} \geq \sum_{k>(a+\delta) K}^{\lfloor(b-\delta) K\rfloor} \operatorname{Pr}\left\{a<\frac{C_{1}}{N} \leq b \mid C_{1}\left(T^{2}\right)=k\right\} \operatorname{Pr}\left\{C_{1}\left(T^{2}\right)=k\right\} .
$$

In order to obtain a lower bound for the right hand side of (2.28), we first define two random variables

$$
W=\left|\left\{T_{K, L}(v): v \in \mathcal{C}_{1}\left(T^{2}\right)\right\}\right| \quad \text { and } \quad S=\left|\left\{T_{K, L}(v): v \in V_{1}\right\}\right|
$$

The key observation is that for $(a+\delta) K<k \leq(b-\delta) K, 1 \leq s \leq K$, and $1 \leq w \leq k$,

$$
\operatorname{Pr}\left\{a N<C_{1} \leq b N \mid W=w, S=s, C_{1}\left(T^{2}\right)=k\right\}=\operatorname{Pr}\{a N<Y+k+w \leq b N\}
$$

where $Y \sim \operatorname{Bin}(L-s, k / K)$. It follows from Chernoff's large deviation bounds for binomial variables (see for example [20] p.39) that for $k \leq(b-\delta) K, 1 \leq s \leq K, 1 \leq w \leq k$ and $K$ large enough,

$$
\begin{aligned}
\operatorname{Pr}\{Y \leq b N-k-w\} & \geq \operatorname{Pr}\{Y \leq b L-2(b-\delta) K\} \geq \operatorname{Pr}\{\tilde{Y} \leq b L-2(b-\delta) K\} \\
& \geq \operatorname{Pr}\{\tilde{Y} \leq(b-\delta) L+\log (K) \cdot \sqrt{(b-\delta)(1-b+\delta) L}\} \\
& \geq 1-\frac{C(b, \delta)}{K}
\end{aligned}
$$

where $\tilde{Y} \sim \operatorname{Bin}(L, b-\delta)$ and $C(b, \delta)$ is a constant which does not depend on $K$. Similarly, for $(a+\delta) K<k, 1 \leq s \leq K$, and $1 \leq w \leq k$, large deviation bounds yield

$$
\begin{aligned}
\operatorname{Pr}\{Y>a N-k-w\} & \geq \operatorname{Pr}\{Y>a N\} \geq \operatorname{Pr}\{\hat{Y}>a N\} \\
& \geq \operatorname{Pr}\{\hat{Y}>(a+\delta)(L-K)-\log (K) \cdot \sqrt{(a+\delta)(1-a-\delta)(L-K)}\} \\
& \geq 1-\frac{C(a, \delta)}{K}
\end{aligned}
$$

for all large $K$, where $\hat{Y} \sim \operatorname{Bin}(L-K, a+\delta)$. So

$$
\operatorname{Pr}\left\{a N<C_{1} \leq b N \mid W=w, S=s, C_{1}\left(T^{2}\right)=k\right\} \geq 1-\frac{C(a, b, \delta)}{K},
$$

for $1 \leq s \leq K, 1 \leq w \leq k$ and hence

$$
\operatorname{Pr}\left\{a<\frac{C_{1}}{N} \leq b \mid C_{1}\left(T^{2}\right)=k\right\} \geq 1-\frac{C(a, b, \delta)}{K}
$$

for all large $K$ and $(a+\delta) K<k \leq(b-\delta) K$. Substituting this bound into (2.28), we obtain

$$
\operatorname{Pr}\left\{a<\frac{C_{1}}{N} \leq b\right\} \geq \operatorname{Pr}\left\{(a+\delta) K<C_{1}\left(T^{2}\right) \leq(b-\delta) K\right\}-\frac{C(a, b, \delta)}{K}
$$


To obtain an upper bound, we note that

$$
\begin{aligned}
\operatorname{Pr}\left\{a<\frac{C_{1}}{N} \leq b\right\} \leq & \operatorname{Pr}\left\{a-\delta<\frac{C_{1}\left(T^{2}\right)}{K} \leq b+\delta\right\}+\operatorname{Pr}\left\{\frac{C_{1}}{N} \leq b, \frac{C_{1}\left(T^{2}\right)}{K}>b+\delta\right\} \\
& +\operatorname{Pr}\left\{\frac{C_{1}\left(T^{2}\right)}{K} \leq a-\delta, \frac{C_{1}}{N}>a\right\} .
\end{aligned}
$$

Large deviation calculations similar to those made above, yield

$\operatorname{Pr}\left\{\frac{C_{1}}{N} \leq b, \frac{C_{1}\left(T^{2}\right)}{K}>b+\delta\right\} \leq \frac{C(b, \delta)}{K}$ and $\operatorname{Pr}\left\{\frac{C_{1}\left(T^{2}\right)}{K} \leq a-\delta, \frac{C_{1}}{N}>a\right\} \leq \frac{C(a, \delta)}{K}$,

for all large $K$. It follows that

$$
\operatorname{Pr}\left\{a<\frac{C_{1}}{N} \leq b\right\} \leq \operatorname{Pr}\left\{a-\delta<\frac{C_{1}\left(T^{2}\right)}{K} \leq b+\delta\right\}+\frac{C(a, b, \delta)}{K} .
$$

So it follows from Lemma 3, the choice of $\delta>0$, and inequalities (2.29) and (2.30) that there exists $K_{\varepsilon}^{\prime \prime}>0$ such that if $K>K_{\varepsilon}^{\prime \prime}$ and $L+L(K) \geq K^{7 / 4}$, then

$$
\left|\operatorname{Pr}\left\{a<\frac{C_{1}}{N} \leq b\right\}-\int_{a}^{b} \frac{d x}{2 \sqrt{1-x}}\right|<\varepsilon .
$$

Thus (2.27) holds for all $K>K_{\varepsilon}=K_{\varepsilon}^{\prime} \vee K_{\varepsilon}^{\prime \prime}$ and $L=L(K) \geq \eta K$ and this completes the proof of the theorem.

\section{ORDER STATISTICS FOR COMPONENT SIZES}

In this section we prove Theorem 2 which gives the limiting distribution of the normalized order statistics for the component sizes of a bipartite random mapping $T_{K, L}$ as $K, L \rightarrow \infty$. Before proving this result, we need some additional notation. We denote by $R_{i}$ the number of red vertices and by $B_{i}$ the number of blue vertices in the $i^{\text {th }}$ connected component $\mathcal{C}_{i}$. Clearly

$$
R_{1}+B_{1}=C_{1}, R_{2}+B_{2}=C_{2}, R_{3}+B_{3}=C_{3}, \ldots
$$

Moreover let $K_{1}=K, L_{1}=L, N_{1}=K_{1}+L_{1}=N$ and for $i \geq 2$

$$
K_{i}=K_{i-1}-R_{i-1} ; \quad L_{i}=L_{i-1}-B_{i-1} ; \quad N_{i}=K_{i}+L_{i}=N-C_{1}-C_{2}-\cdots-C_{i-1}
$$

and note that for $i \geq 2, K_{i}, L_{i}, N_{i}$ are random variables. With this notation we have $X_{i}=C_{i} / N_{i}$. We now proceed to prove Theorem 2 .

Proof of Theorem 2. Observe that by symmetry it suffices to prove the result in the case $L=L(K) \geq K$. Now by the convergence principle outlined in Section 1 , it is enough to show that for any $t \geq 1$ and any $0<a_{i}<b_{i}<1, i=1,2, \ldots, t$,

$$
\lim _{K \rightarrow \infty} \operatorname{Pr}\left\{a_{i}<X_{i} \leq b_{i}, \quad i=1,2, \ldots, t\right\}=\prod_{i=1}^{t} \int_{a_{i}}^{b_{i}} \frac{d u}{\sqrt{1-u}} .
$$


To establish (3.1) we divide the proof into two cases.

Case 1. Suppose that for each $K>0$ we have $K \leq L(K) \leq K^{3}$. For conciseness, we introduce

$$
\mathcal{A}_{j}=\left\{a_{i}<X_{i} \leq b_{i}, \quad i=1,2, \ldots, j\right\} \quad \text { for } \quad j=1,2, \ldots, t,
$$

and we write

$$
\operatorname{Pr}\left\{a_{i}<X_{i} \leq b_{i}, \quad i=1,2, \ldots, t\right\}=\operatorname{Pr}\left\{\mathcal{A}_{t}\right\}=\operatorname{Pr}\left\{\mathcal{B}_{t} \cap \mathcal{A}_{t}\right\}+\operatorname{Pr}\left\{\mathcal{B}_{t}^{c} \cap \mathcal{A}_{t}\right\}
$$

where

$$
\mathcal{B}_{1}=\left\{L_{1} \geq K_{1}\right\}
$$

and for $j=2, \ldots, t$

$$
\mathcal{B}_{j}=\left\{L_{1} \geq K_{1}, \quad \frac{1}{2^{i}} \leq \frac{L_{i+1}}{K_{i+1}}, \quad K \cdot \prod_{s=1}^{i}\left(1-b_{s}-\delta\right) \leq K_{i+1}, \quad i=1,2, \ldots, j-1\right\}
$$

with $\delta:=\frac{1}{2} \min \left\{\left(1-b_{i}\right): i=1,2, \ldots, t\right\}$

Observe that

$$
\begin{aligned}
\operatorname{Pr}\left\{\mathcal{B}_{t} \cap \mathcal{A}_{t}\right\}= & \prod_{j=1}^{t-1} \operatorname{Pr}\left\{\frac{1}{2^{j}} \leq \frac{L_{j+1}}{K_{j+1}}, K \cdot \prod_{s=1}^{j}\left(1-b_{s}-\delta\right) \leq K_{j+1} \mid \mathcal{B}_{j} \cap \mathcal{A}_{j}\right\} \\
& \times \prod_{i=1}^{t} \operatorname{Pr}\left\{a_{i}<X_{i} \leq b_{i} \mid \mathcal{B}_{i} \cap \mathcal{A}_{i-1}\right\}
\end{aligned}
$$

where $\mathcal{B}_{1} \cap \mathcal{A}_{0}:=\mathcal{B}_{1}$. The first step is to show that

$$
\lim _{K \rightarrow \infty} \prod_{i=1}^{t} \operatorname{Pr}\left\{a_{i}<X_{i} \leq b_{i} \mid \mathcal{B}_{i} \cap \mathcal{A}_{i-1}\right\}=\prod_{i=1}^{t} \int_{a_{i}}^{b_{i}} \frac{d u}{2 \sqrt{1-u}}
$$

Note that by conditioning on the events $\mathcal{B}_{i}$ in the terms in the product (3.4) we guarantee that for each $1 \leq i \leq t, K_{i} \rightarrow \infty$ as $K \rightarrow \infty$ and $\frac{1}{2^{i-1}} K_{i} \leq L_{i}$. So by Theorem 1 , for $i=1$,

$$
\lim _{K \rightarrow \infty} \operatorname{Pr}\left\{a_{1}<X_{1} \leq b_{1} \mid 1 \leq \frac{L}{K}\right\}=\lim _{K, L \rightarrow \infty} \operatorname{Pr}\left\{a_{1}<X_{1} \leq b_{1}\right\}=\int_{a_{1}}^{b_{1}} \frac{d u}{\sqrt{1-u}}
$$

For $2 \leq i \leq t$ we exploit the identity

$$
\operatorname{Pr}\left\{a_{i}<X_{i} \leq b_{i} \mid K_{i}=r, L_{i}=b, \mathcal{B}_{i-1} \cap \mathcal{A}_{i-1}\right\}=\operatorname{Pr}\left\{a_{i}<\frac{C_{1}(r, b)}{r+b} \leq b_{i}\right\}
$$

where $C_{1}(r, b)$ is the size of the component which contains the vertex 1 in a random bipartite mapping on $r$ red vertices and $b$ blue vertices. This identity is a straightforward consequence of the independence and uniformity which is built into our model, namely, that each vertex is assigned independently, according to the uniform distribution, to a 
vertex in the other set. So by Theorem 1 , for each $2 \leq i \leq t$, there exists $K(\varepsilon, i)>0$ such that if $K>K(\varepsilon, i), K \prod_{s=1}^{i-1}\left(1-b_{s}-\delta\right) \leq r<K$ and $\frac{r}{2^{i-1}} \leq b<L$, then

$$
\begin{gathered}
\left|\operatorname{Pr}\left\{a_{i}<X_{i} \leq b_{i} \mid K_{i}=r, L_{i}=b, \mathcal{B}_{i-1} \cap \mathcal{A}_{i-1}\right\}-\int_{a_{i}}^{b_{i}} \frac{d u}{2 \sqrt{1-u}}\right| \\
=\left|\operatorname{Pr}\left\{a_{i}<\frac{C_{1}(r, b)}{r+b} \leq b_{i}\right\}-\int_{a_{i}}^{b_{i}} \frac{d u}{2 \sqrt{1-u}}\right| \leq \varepsilon
\end{gathered}
$$

It follows that if $K>\max \{K(\varepsilon, i): i=1,2, \ldots, t\}$ then

$$
\left|\operatorname{Pr}\left\{a_{i}<X_{i} \leq b_{i} \mid \mathcal{B}_{i} \cap \mathcal{A}_{i-1}\right\}-\int_{a_{i}}^{b_{i}} \frac{d u}{2 \sqrt{1-u}}\right|<\varepsilon \quad \text { for } 2 \leq i \leq t
$$

and (3.4) now follows from (3.5) and (3.7). Next we show that the events $\mathcal{B}_{i}$ on which we have conditioned in the calculations above (and which guarantee that we can apply Theorem 1) have high probability. In particular, we claim that

$$
\lim _{K \rightarrow \infty} \prod_{j=1}^{t-1} \operatorname{Pr}\left\{\frac{1}{2^{j}} \leq \frac{L_{j+1}}{K_{j+1}}, K \prod_{s=1}^{j}\left(1-b_{s}-\delta\right) \leq K_{j+1} \mid \mathcal{B}_{j} \cap \mathcal{A}_{j}\right\}=1 .
$$

Now given $\mathcal{B}_{j} \cap \mathcal{A}_{j}$, we have for $1 \leq j \leq t-1$

$$
\frac{L_{j+1}}{K_{j+1}}=\frac{L_{j}-\left(L_{j} X_{j}-d_{j}\right)}{K_{j}-\left(K_{j} X_{j}+d_{j}\right)}=\frac{L_{j}}{K_{j}} \cdot \frac{1+d_{j} /\left(L_{j}\left(1-X_{j}\right)\right)}{1-d_{j} /\left(K_{j}\left(1-X_{j}\right)\right)} \geq \frac{1}{2^{j-1}} \cdot \frac{1+d_{j} /\left(L_{j}\left(1-X_{j}\right)\right)}{1-d_{j} /\left(K_{j}\left(1-X_{j}\right)\right)}
$$

where $d_{j}=L_{j} X_{j}-B_{j}=R_{j}-K_{j} X_{j}$, and

$$
K_{j+1}=K_{j}-K_{j} X_{j}-d_{j} \geq K_{j}\left(1-b_{j}-\frac{d_{j}}{K_{j}}\right) \geq\left(1-b_{j}-\frac{d_{j}}{K_{j}}\right) \cdot K \prod_{s=1}^{j-1}\left(1-b_{s}-\delta\right)
$$

(with the convention that for $j=1$ the product in the above formula is equal to 1 ). Hence

$$
\begin{aligned}
& \operatorname{Pr}\left\{\frac{1}{2^{j}} \leq \frac{L_{j+1}}{K_{j+1}}, K \cdot \prod_{s=1}^{j}\left(1-b_{s}-\delta\right) \leq K_{j+1} \mid \mathcal{B}_{j} \cap \mathcal{A}_{j}\right\} \\
& \geq \operatorname{Pr}\left\{\frac{1}{2} \leq \frac{1+d_{j} / L_{j}\left(1-X_{j}\right)}{1-d_{j} / K_{j}\left(1-X_{j}\right)}, \frac{\left|d_{j}\right|}{K_{j}} \leq \delta \mid \mathcal{B}_{j} \cap \mathcal{A}_{j}\right\} .
\end{aligned}
$$

Also, given the event $\mathcal{B}_{j} \cap \mathcal{A}_{j}$, we have $L_{j} \geq K_{j} / 2^{j-1}$ and $K_{j} \geq K \prod_{s=1}^{j-1}\left(1-b_{s}-\delta\right)$, so if $\left|d_{j}\right| \leq\left(K_{j}\right)^{2 / 3}$, then

$$
\frac{\left|d_{j}\right|}{L_{j}\left(1-X_{j}\right)} \leq \frac{C(j)}{K^{1 / 3}} \quad \text { and } \quad \frac{\left|d_{j}\right|}{K_{j}} \leq \frac{\left|d_{j}\right|}{K_{j}\left(1-X_{j}\right)} \leq \frac{C(j)}{K^{1 / 3}}
$$


where $C(j)$ is a constant which depends on $j, b_{1}, b_{2}, \ldots, b_{j}$. Therefore, given $\mathcal{B}_{j} \cap \mathcal{A}_{j}$, if $\left|d_{j}\right| \leq\left(K_{j}\right)^{2 / 3}$, then

$$
\frac{1}{2} \leq \frac{1+d_{j} / L_{j}\left(1-X_{j}\right)}{1-d_{j} / K_{j}\left(1-X_{j}\right)} \quad \text { and } \quad \frac{\left|d_{j}\right|}{K_{j}}<\delta
$$

and

$$
\operatorname{Pr}\left\{\frac{1}{2} \leq \frac{1+d_{j} / L_{j}\left(1-X_{j}\right)}{1-d_{j} / K_{j}\left(1-X_{j}\right)}, \frac{\left|d_{j}\right|}{K_{j}} \leq \delta \mid \mathcal{B}_{j} \cap \mathcal{A}_{j}\right\} \geq \operatorname{Pr}\left\{\left|d_{j}\right| \leq\left(K_{j}\right)^{2 / 3} \mid \mathcal{B}_{j} \cap \mathcal{A}_{j}\right\}
$$

for all $1 \leq j \leq t-1$ and all sufficiently large $K$.

In the remaining calculations we make use of the identity

$$
\operatorname{Pr}\left\{R_{j}=k, B_{j}=l \mid K_{j}=r, L_{j}=b, \mathcal{B}_{j-1} \cap \mathcal{A}_{j-1}\right\}=\operatorname{Pr}\left\{R_{1}(r, b)=k, B_{1}(r, b)=l\right\}
$$

where $R_{1}(r, b)$ is the number of red vertices and $B_{1}(r, b)$ is the number of blue vertices in the connected component containing the vertex " 1 " in $G\left(T_{r, b}\right)$, the digraph which represents the bipartite random mapping $T_{r, b}$ on $r$ red and $b$ blue vertices. In particular, for $r, b$ and $m$ chosen such that $K \prod_{s=1}^{j-1}\left(1-b_{s}-\delta\right) \leq r<K, r / 2^{j-1} \leq b<L$, and $a_{j}<x=m /(r+b) \leq b_{j}$,

$$
\begin{aligned}
& \operatorname{Pr}\left\{\left|d_{j}\right|>r^{2 / 3}, C_{j}=m \mid K_{j}=r, L_{j}=b, \mathcal{B}_{j-1} \cap \mathcal{A}_{j-1}\right\} \\
\leq & \sum_{|i|>r^{2 / 3}-1} \operatorname{Pr}\left\{R_{1}(r, b)=[r x]-i, B_{1}(r, b)=[b x]+i\right\}
\end{aligned}
$$

$$
\leq \sum_{\left\{k:|[r x]-k-1|>r^{2 / 3}-1\right\}}\left(\begin{array}{c}
r-1 \\
k
\end{array}\right)\left(\frac{m-k-1}{b}\right)^{k}\left(1-\frac{m-k-1}{b}\right)^{r-k-1}
$$

where

$$
[r x]+[b x]=m \quad \text { and } \quad|[r x]-r x| \leq 1, \quad|[b x]-b x| \leq 1 .
$$

We note that inequality (3.11) follows from an argument similar to the reasoning which we used to establish inequality (2.11). The right hand side of (3.11) is a 'two-sided' sum. We indicate how to bound one side of the sum. Note that

$$
\begin{aligned}
& \sum_{k>[r x]+r^{2 / 3}-2}\left(\begin{array}{c}
r-1 \\
k
\end{array}\right)\left(\frac{m-k-1}{b}\right)^{k}\left(1-\frac{m-k-1}{b}\right)^{r-k-1} \\
\leq & \sum_{k>[r x]+r^{2 / 3}-2}\left(\begin{array}{c}
r-1 \\
k
\end{array}\right)\left(\tilde{x}-\frac{r^{2 / 3}-1}{b}\right)^{k}\left(1-\tilde{x}+\frac{r^{2 / 3}-1}{b}\right)^{r-k-1} \\
\leq & \operatorname{Pr}\left\{\frac{X-E(X)}{\sqrt{\operatorname{Var}(X)}}>\frac{r^{2 / 3}-1}{\sqrt{\operatorname{Var}(X)}}\right\} \leq \hat{C}(t) \exp \left(-\hat{C}(t) K^{1 / 6}\right)
\end{aligned}
$$

where $\tilde{x}=[b x] / b, X \sim \operatorname{Bin}\left(r-1, \tilde{x}-\frac{r^{2 / 3}-1}{b}\right)$, and $\hat{C}(t)$ is a constant which only depends on $a_{1}, a_{2}, \ldots, a_{t}$ and $b_{1}, b_{2}, \ldots, b_{t}$. The last inequality follows from Chernoff's large deviation 
bound for the binomial distribution and from the assumption that $r / 2^{j-1} \leq b<L$ and $K \prod_{s=1}^{j-1}\left(1-b_{s}-\delta\right) \leq r<K$. Similar calculations yield

$$
\sum_{k<[r x]-r^{2 / 3}}\left(\begin{array}{c}
r-1 \\
k
\end{array}\right)\left(\frac{m-k-1}{b}\right)^{k}\left(1-\frac{m-k-1}{b}\right)^{r-k-1} \leq \hat{C}(t) \exp \left(-\hat{C}(t) K^{1 / 6}\right) .
$$

Since these bounds are uniform over all $r, b$, and $m$ satisfying $K \prod_{s=1}^{j-1}\left(1-b_{s}-\delta\right) \leq r<K$, $r / 2^{j-1} \leq b<L$, and $a_{j}<x=m /(r+b) \leq b_{j}$, we have

$$
\begin{aligned}
& \operatorname{Pr}\left\{\left|d_{j}\right|>r^{2 / 3}, a_{j}<X_{j} \leq b_{j} \mid K_{j}=r, L_{j}=b, \mathcal{B}_{j-1} \cap \mathcal{A}_{j-1}\right\} \\
& =\sum_{m>a_{j}(r+b)}^{b_{j}(r+b)} \operatorname{Pr}\left\{\left|d_{j}\right|>r^{2 / 3}, C_{j}=m \mid K_{j}=r, L_{j}=b, \mathcal{B}_{j-1} \cap \mathcal{A}_{j-1}\right\} \\
& \leq(r+b) \hat{C}(t) \exp \left(-\hat{C}(t) K^{1 / 6}\right) \leq 2 \hat{C}(t) K^{3} \exp \left(-\hat{C}(t) K^{1 / 6}\right) .
\end{aligned}
$$

It follows from this inequality and inequality (3.6) that

$$
\begin{aligned}
& \operatorname{Pr}\left\{\left|d_{j}\right|>r^{2 / 3} \mid K_{j}=r, L_{j}=b, \mathcal{B}_{j-1} \cap \mathcal{A}_{j}\right\} \\
& =\frac{\operatorname{Pr}\left\{\left|d_{j}\right|>r^{2 / 3}, a_{j}<X_{j} \leq b_{j} \mid K_{j}=r, L_{j}=b, \mathcal{B}_{j-1} \cap \mathcal{A}_{j-1}\right\}}{\operatorname{Pr}\left\{a_{j}<X_{j} \leq b_{j} \mid K_{j}=r, L_{j}=b, \mathcal{B}_{j-1} \cap \mathcal{A}_{j-1}\right\}} \leq \frac{\hat{C}(t)}{K}
\end{aligned}
$$

for $1 \leq j \leq t-1$ and all suficiently large $K$, provided $K \prod_{s=1}^{j-1}\left(1-b_{s}-\delta\right) \leq r<K$, and $r / 2^{j-1} \leq b<L$. Hence

$$
\operatorname{Pr}\left\{\left|d_{j}\right| \leq K_{j}^{2 / 3} \mid \mathcal{B}_{j} \cap \mathcal{A}_{j}\right\} \geq 1-\frac{\hat{C}(t)}{K}
$$

for all $1 \leq j \leq t-1$ and all sufficiently large $K$. Equation (3.8) now follows from (3.9), (3.10), and (3.12). Finally we obtain

$$
\lim _{K \rightarrow \infty} \operatorname{Pr}\left\{\mathcal{B}_{t} \cap \mathcal{A}_{t}\right\}=\prod_{i=1}^{t} \int_{a_{1}}^{b_{i}} \frac{d u}{2 \sqrt{1-u}}
$$

from $(3.3),(3.4)$, and (3.8). It remains to show that

$$
\lim _{K \rightarrow \infty} \operatorname{Pr}\left\{\mathcal{B}_{t}^{c} \cap \mathcal{A}_{t}\right\}=0
$$

Observe that

$$
\operatorname{Pr}\left\{\mathcal{B}_{t}^{c} \cap \mathcal{A}_{t}\right\} \leq \sum_{j=1}^{t-1} \operatorname{Pr}\left\{\frac{L_{j+1}}{K_{j+1}}<\frac{1}{2^{j}} \text { or } K_{j+1}<K \prod_{s=1}^{j}\left(1-b_{s}-\delta\right) \mid \mathcal{B}_{j} \cap \mathcal{A}_{j}\right\} \operatorname{Pr}\left\{\mathcal{B}_{j} \cap \mathcal{A}_{j}\right\},
$$

and (3.14) follows immediately from (3.8). Equation (3.1) now follows from (3.2), (3.13) and (3.14). 
Case 2: Now suppose that for each $K>0$, we have $L=L(K) \geq K^{3}$. In this case we take an indirect approach. As in Section 2, $T^{2}=T_{K, L} \circ T_{K, L}, T_{K}$ denotes the uniform random mapping of $V_{1}$ into $V_{1}$, and $G\left(T^{2}\right)$ and $G\left(T_{K}\right)$ denote the random digraphs on $K$ vertices which represent the random mappings $T^{2}$ and $T_{K}$, respectively. We also extend our notation as follows. For any mapping $f$ from $V_{1}$ into $V_{1}$, let $\mathcal{C}_{1}(f)$ denote the component in $G(f)$ which contains the vertex labelled 1 . If $\mathcal{C}_{1}(f) \neq G(f)$, then let $\mathcal{C}_{2}(f)$ denote the component in $G(f) \backslash \mathcal{C}_{1}(f)$ which contains the smallest vertex; otherwise, set $\mathcal{C}_{2}(f)=\emptyset$. For $t>2$ we define $\mathcal{C}_{t}(f)$ iteratively: If $G(f) \backslash\left(\mathcal{C}_{1}(f) \cup \ldots \cup \mathcal{C}_{t-1}(f)\right) \neq \emptyset$, then let $\mathcal{C}_{t}(f)$ denote the component in $G(f) \backslash\left(\mathcal{C}_{1}(f) \cup \ldots \cup \mathcal{C}_{t-1}(f)\right)$ which contains the smallest vertex; otherwise, set $\mathcal{C}_{t}(f)=\emptyset$. For $t \geq 1$, let $C_{t}(f)=\left|\mathcal{C}_{t}(f)\right|$ and define the sequence $\left(X_{1}(f), X_{2}(f), \ldots\right)$ by

$$
X_{1}(f)=\frac{C_{1}(f)}{K_{1}(f)}, X_{2}(f)=\frac{C_{2}(f)}{K_{2}(f)}, \ldots, X_{t}(f)=\frac{C_{t}(f)}{K_{t}(f)}, \ldots
$$

where $K_{1}(f)=K, K_{i}(f)=K_{i-1}(f)-C_{i-1}(f)=K-C_{1}(f)-C_{2}(f)-\ldots-C_{i-1}(f)$ for $i \geq 2$, and $X_{i}(f)=0$ if $K_{i}(f)=0$.

Now let

$$
\mathcal{H}_{K}=\left\{T_{K, L}(v) \neq T_{K, L}(w) \text { for all } v, w \in V_{1}, v \neq w\right\},
$$

then it is easy to show that given $\mathcal{H}_{K}$, the random mapping $T^{2}$ on $V_{1}$ has the same distribution as the uniform random mapping $T_{K}$. In particular, for any $t \geq 1$ and $0<a_{i}<b_{i}<1, i=1,2, \ldots, t$,

$$
\operatorname{Pr}\left\{a_{i}<X_{i}\left(T^{2}\right) \leq b_{i}: i=1,2, \ldots, t \mid \mathcal{H}_{K}\right\}=\operatorname{Pr}\left\{a_{i}<X_{i}\left(T_{K}\right) \leq b_{i}: i=1,2, \ldots, t\right\} .
$$

Furthermore, provided $L=L(K) \geq K^{3}$,

$$
\operatorname{Pr}\left\{\mathcal{H}_{K}\right\}=\frac{(L)_{K}}{L^{K}} \geq 1-\frac{C}{K}
$$

where $C$ is a constant which does not depend on $K$. It follows from (3.15) and (3.16) that for any $t \geq 1$ and $0<a_{i}<b_{i}<1, i=1,2, \ldots, t$,

$$
\begin{gathered}
\operatorname{Pr}\left\{a_{i}<X_{i}\left(T_{K}\right) \leq b_{i}: i=1,2, \ldots, t\right\}\left(1-\frac{C}{K}\right) \leq \operatorname{Pr}\left\{a_{i}<X_{i}\left(T^{2}\right) \leq b_{i}: i=1,2, \ldots, t\right\} \\
\leq \operatorname{Pr}\left\{a_{i}<X_{i}\left(T_{K}\right) \leq b_{i}: i=1,2, \ldots, t\right\}+\frac{C}{K}
\end{gathered}
$$

For the uniform random mapping $T_{K}$ (see [1])

$$
\lim _{K \rightarrow \infty} \operatorname{Pr}\left\{a_{i}<X_{i}\left(T_{K}\right) \leq b_{i}: i=1,2, \ldots, t\right\}=\prod_{i=1}^{t} \int_{a_{i}}^{b_{i}} \frac{d u}{2 \sqrt{1-u}}
$$

so it follows that

$$
\lim _{K \rightarrow \infty} \operatorname{Pr}\left\{a_{i}<X_{i}\left(T^{2}\right) \leq b_{i}: i=1,2, \ldots, t\right\}=\prod_{i=1}^{t} \int_{a_{i}}^{b_{i}} \frac{d u}{2 \sqrt{1-u}} .
$$


Thus, to obtain (3.1) in the case when $L=L(K) \geq K^{3}$ it suffices to show that for every $t \geq 1$ and every $\varepsilon>0$,

$$
\lim _{K \rightarrow \infty} \operatorname{Pr}\left\{\left|X_{i}\left(T^{2}\right)-X_{i}\right|<\varepsilon: i=1,2, \ldots, t\right\}=1
$$

Fix $t \geq 1, \varepsilon>0$, and $\delta>0$ and choose $\rho=\rho(\delta)>0$ such that

$$
\left(\int_{\rho}^{1-\rho} \frac{d u}{2 \sqrt{1-u}}\right)^{t}>1-\delta
$$

and let

$$
\mathcal{A}_{K}(\rho)=\left\{\rho \leq X_{i}\left(T^{2}\right) \leq 1-\rho: i=1,2, \ldots, t\right\} .
$$

We note that if $\rho \leq X_{i}\left(T^{2}\right) \leq 1-\rho$ for $i=1,2, \ldots, t$, then by induction

$$
\rho^{i-1} K \leq K_{i} \leq(1-\rho)^{i-1} K \quad \text { and } \quad \rho^{i} K \leq C_{i}\left(T^{2}\right) \leq(1-\rho)^{i} K
$$

for $1 \leq i \leq t$. Also, it follows from (3.15) and (3.17) that

$$
\lim _{K \rightarrow \infty} \operatorname{Pr}\left\{\mathcal{A}_{K}(\rho) \mid \mathcal{H}_{K}\right\}=\left(\int_{\rho}^{1-\rho} \frac{d u}{2 \sqrt{1-u}}\right)^{t}>1-\delta
$$

and hence

$$
\begin{gathered}
\limsup _{K \rightarrow \infty} \operatorname{Pr}\left\{\left|X_{i}\left(T^{2}\right)-X_{i}\right|<\varepsilon: i=1,2, \ldots, t\right\} \\
\geq \limsup _{K \rightarrow \infty} \operatorname{Pr}\left\{\left|X_{i}\left(T^{2}\right)-X_{i}\right|<\varepsilon, i=1,2, \ldots, t \mid \mathcal{A}_{K}(\rho), \mathcal{H}_{K}\right\} \operatorname{Pr}\left\{\mathcal{A}_{K}(\rho) \mid \mathcal{H}_{K}\right\} \operatorname{Pr}\left\{\mathcal{H}_{K}\right\} \\
(3.21)>\limsup _{K \rightarrow \infty} \operatorname{Pr}\left\{\left|X_{i}\left(T^{2}\right)-X_{i}\right|<\varepsilon, i=1,2, \ldots, t \mid \mathcal{A}_{K}(\rho), \mathcal{H}_{K}\right\}(1-\delta) .
\end{gathered}
$$

We claim that

$$
\limsup _{K \rightarrow \infty} \operatorname{Pr}\left\{\left|X_{i}\left(T^{2}\right)-X_{i}\right|<\varepsilon, i=1,2, \ldots, t \mid \mathcal{A}_{K}(\rho), \mathcal{H}_{K}\right\}=1
$$

To prove (3.22) we define variables $Y_{i}=C_{i}-2 C_{i}\left(T^{2}\right)$ for $1 \leq i \leq t$. Using this notation, we have

$$
X_{i}=\frac{2 C_{i}\left(T^{2}\right) / L+Y_{i} / L}{1+K / L-2\left(C_{1}\left(T^{2}\right)+\ldots+C_{i-1}\left(T^{2}\right)\right) / L-Y_{1} / L-Y_{2} / L-\ldots-Y_{i-1} / L}
$$

and

$$
X_{i}\left(T^{2}\right)=\frac{C_{i}\left(T^{2}\right) / K}{1-C_{1}\left(T^{2}\right) / K-\ldots-C_{i-1}\left(T^{2}\right) / K} .
$$

So if $L=L(K) \geq K^{3}, \rho \leq X_{i}\left(T^{2}\right) \leq 1-\rho$, and

$$
\left|\frac{Y_{i}}{L}-\frac{C_{i}\left(T^{2}\right)}{K}\right|<\frac{2}{L^{1 / 3}}<\frac{2}{K} \quad \text { for } 1 \leq i \leq t,
$$


then it follows from these inequalitites and from (3.20) that

$$
\left|X_{i}-X_{i}\left(T^{2}\right)\right|<\frac{C(\rho, t)}{K} \quad \text { for } 1 \leq i \leq t,
$$

where $C(\rho, t)$ is a constant which depends only on $\rho$ and $t$. Hence

$$
\begin{gathered}
\operatorname{Pr}\left\{\left|X_{i}\left(T^{2}\right)-X_{i}\right|<\varepsilon, i=1,2, \ldots, t \mid \mathcal{A}_{K}(\rho), \mathcal{H}_{K}\right\} \\
\geq \operatorname{Pr}\left\{\left|\frac{Y_{i}}{L}-\frac{C_{i}\left(T^{2}\right)}{K}\right|<\frac{2}{L^{1 / 3}}, i=1,2, \ldots, t \mid \mathcal{A}_{K}(\rho), \mathcal{H}_{K}\right\}
\end{gathered}
$$

for all sufficiently large $K$.

Now fix $K>0$ and integers $0<r_{1}, r_{2}, \ldots, r_{t}<K$ such that if $C_{i}\left(T^{2}\right)=r_{i}$ for $1 \leq i \leq t$, then $\rho \leq X_{i}\left(T^{2}\right) \leq 1-\rho$ for $1 \leq i \leq t$, i.e.,

$$
\rho \leq X_{i}\left(T^{2}\right)=\frac{r_{i} / K}{1-r_{1} / K-r_{2} / K-\ldots-r_{i-1} / K} \leq 1-\rho
$$

for every $i=1,2, \ldots, t$. Now given $\mathcal{H}_{K}$ and $C_{i}\left(T^{2}\right)=r_{i}$ for $1 \leq i \leq t$, we have

$$
C_{i}=2 r_{i}+Y_{i} \quad \text { for } \quad 1 \leq i \leq t
$$

where the marginal distribution of each $Y_{i}$ is $\operatorname{Bin}\left(L-r_{i}, \frac{r_{i}}{K}\right)$. It follows from Chebyshev's inequality that

$$
\begin{aligned}
& \operatorname{Pr}\left\{\left|\frac{Y_{i}}{L}-\frac{r_{i}}{K}\right|<\frac{2}{L^{1 / 3}}, i=1,2, \ldots, t \mid C_{i}\left(T^{2}\right)=r_{i}, i=1,2, \ldots, t, \mathcal{H}_{K}\right\} \\
& \geq \operatorname{Pr}\left\{\left|Y_{i}-\frac{L r_{i}}{K}-\frac{r_{i}^{2}}{K}\right|<L^{2 / 3}, i=1,2, \ldots, t \mid C_{i}\left(T^{2}\right)=r_{i}, i=1,2, \ldots, t, \mathcal{H}_{K}\right\} \\
& \geq 1-\sum_{i=1}^{t} \frac{\left(L-r_{i}\right)}{4 L^{4 / 3}} \geq 1-\frac{t}{4 L^{1 / 3}} \geq 1-\frac{t}{4 K} .
\end{aligned}
$$

Since this inequality holds for all $0<r_{1}, r_{2}, \ldots, r_{t}<K$ satisfying (3.24), we obtain

$$
\operatorname{Pr}\left\{\left|\frac{Y_{i}}{L}-\frac{C_{i}\left(T^{2}\right)}{K}\right|<\frac{2}{L^{1 / 3}}, i=1,2, \ldots, t \mid \mathcal{A}_{K}(\rho), \mathcal{H}_{K}\right\} \geq 1-\frac{t}{4 K} .
$$

Equation (3.22) now follows from (3.23) and (3.25) and we obtain

$$
\limsup _{K \rightarrow \infty} \operatorname{Pr}\left\{\left|X_{i}\left(T^{2}\right)-X_{i}\right|<\varepsilon: i=1,2, \ldots, t\right\}>1-\delta
$$

from (3.21) and (3.22). Letting $\delta \downarrow 0$, we obtain (3.19) and the theorem is proved in this case.

It follows from Case 1 and Case 2, that (3.1) holds under the hypotheses of the theorem and the proof is complete. 


\section{ACKNOWLEDGEMENTS}

The authors would like to thank their respective departments for supporting the research visits during which this paper was written. We would also like to thank the referees for their careful reading of this paper and their suggestions which have improved the exposition of this paper.

\section{REFERENCES}

[1] D. Aldous, Exchangeability and related topics, Lecture Notes in Mathematics, 1117, Springer Verlag, New York, 1985.

[2] R. Arratia and S. Tavaré, The cycle structure of random permutations, Ann. Probab., 20, 1567-1591 (1992).

[3] R. Arratia and S. Tavaré, Limit theorems for combinatorial structures via discrete process approximations, Random Structures and Algorithms, 3, 321-345 (1992).

[4] R. Arratia, D. Stark and S. Tavaré, Total variation asymptotics for Poisson process approximations of logarithmic combinatorial assemblies, Ann. Probab., 23, 1347 -1388 (1995).

[5] T. L. Austin, The enumeration of point labelled chromatic graphs and trees, Canadian Journal of Mathematics, 12, 535-545 (1960).

[6] B. Bollobás, Random Graphs, Academic Press, London, 1985.

[7] Y. D. Burtin, On simple formula for random mappings and its applications, J. Appl. Probab. , 17, 403-414 (1980).

[8] J. M. DeLaurentis and B. Pittel, Random permutations and Brownian motion,Pacific J. Math., 119, 287-301 (1985).

[9] W. Feller, An Introduction to Probability Theory and its Applications, Vol I , 3rd ed., John Wiley and Sons, New York, 1970.

[10] J. C. Hansen, A functional central limit theorem for random mappings, Ann. of Probab., 17, 317-332 (1989).

[11] J. C. Hansen, Order statistics for decomposable combinatorial structures, Random Structures and Algorithms, 5, 517-533 (1994).

[12] J. Jaworski, On the connectedness of a random bipartite mapping, Graph Theory, Eagów 1981, Lecture Notes in Mathematics, 1018, 69-74, Springer Verlag, New York, 1983.

[13] J. Jaworski, A random bipartite mapping, Annals of Discrete Mathematics, 28, 137-158 (1985).

[14] J. Jaworski, Predecessors in a random mapping, Random Structures \& Algorithms, 13, No.3 and No.4, 501-519 (1998).

[15] J. Jaworski, Epidemic processes on digraphs of random mappings, J. Appl. Probab., 36, 780-798 (1999).

[16] J. Jaworski and M. Karoński, On the connectivity of graphs generated by a sum of random mappings, Journal of Graph Theory, 17, No.2, 135-150 (1993).

[17] J. Jaworski and T. Łuczak, Cycles in a uniform graph process, Combinatorics, Probability and Computing, 1, 223-239 (1992).

[18] V. F. Kolchin, Random Mappings, Optimization Software Inc., New York, 1986.

[19] T. Euczak, On the equivalence of two basic models of a random graph, in: M. Karoński, J. Jaworski, A. Ruciński (eds.): Random Graphs '87. John Wiley \& Sons, New York

- Chichester - Brisbane 1990, pp. 151-158. 
[20] S. M. Ross, Stochastic Processes, 2nd edition, John Wiley and Sons, New York, 1996.

[21] V. E. Stepanov, Random mappings with a single attracting centre, Theory Prob. Appl. 16, 155-161 (1971).

[22] A.M. Vershik and A.A. Shmidt, Limit measures arising in the asymptotic theory of symmetric groups. I, Theor. Probab. Appl., 22, 70-85 (1977). 